\title{
Design the Middleware of Intelligent Monitoring System for Vehicles
}

\author{
Hwase Park ${ }^{1}$, Seokil Song ${ }^{2}$, Youngsik Kwak ${ }^{3}$ and Yoonsik Kwak ${ }^{4 *}$ \\ ${ }^{1}$ Daelim Collage University \\ ${ }^{2,4}$ Dept. of Computer. Eng. Korea National University of Transportation \\ ${ }^{3}$ Gyeongnam National University of Science and Technology \\ ${ }^{1}$ hwasepark@gmail.com, ${ }^{2,4}\left\{\right.$ sisong,yskwak\}@ut.ac.kr, ${ }^{3}$ yskwak@gntech.ac.kr
}

\begin{abstract}
This paper presents an intelligent monitoring system for the economical driving of vehicles. It is about the design and implementation of a framework to provide intelligent monitoring services for vehicles based on cloud and $4 G$ LTE technologies. A large amount of data, such as the information of driving, the status of vehicles, moving and still images collected by LTE devices in the vehicles, is stored and managed by the proposed monitoring framework for vehicles in a distributed cloud environment. An API with effective indexing and analyzing functions based on MapReduce are provided. The proposed system can be used to quickly design and implement an application system based on a user's needs.
\end{abstract}

Keywords: Monitoring system, Vehicles, Sensor Networks, Middleware

\section{Introduction}

This paper is about the design and implementation of an intelligent monitoring system to support economical and safe driving using the convergence of IT and vehicle technologies. The proposed system use information collected from terminal devices based on $4 \mathrm{G}$, and has functions to store and manipulate those data. Due to advances in information communication and computer technologies, the needs for ubiquitous systems in various fields have been increased. User's needs for new technologies and services are increasing beyond location tracking systems to simply show the location information of vehicles on screen. As an example, information about the current location as well as the past driving path of vehicles are stored, and needs for technologies to manipulate a user's time-space queries based on location information have increased. Based on such technologies and stored information, research to detect useful driving patterns and provide effective driving information about vehicles is being actively performed.[1-3]

As another example, if navigation devices are fitted with a taxi-call program and wireless modem ( $3 \mathrm{G} / \mathrm{WiBro}$ ), and the location of taxis could be periodically tracked based on GPS, it would be possible to provide various services. When a request for a taxi-call occurs, it is recorded at a monitoring center. Next, after searching the location information of taxis in real time, the transferring of messages to a taxi in the best conditions is processed by the monitoring system.

BIS/BMS(Bus Information System/Bus Management System) is an advanced traffic monitoring system to provide driving information in real time. Terminal devices of the BMS are equipped in buses, which are also equipped with GPS/wireless modems. When events happen during driving (regarding current location information, time, speed, etc.), information about events is transferred to a traffic information center over wireless internet. The data can be used for services of overall driving management such as

*Corresponding Author 
providing information about departure/arrivals of buses, controlling the time interval between buses, providing information about the current locations of buses, providing information about accidents / failures, and so on.

For vehicle monitoring services for express buses, automatically information about driving (status information of vehicles regarding speed, brakes, RPM, mileage, traffic accidents, and so on) is accumulated in a digital tachograph (DTG) file. Through providing a screen based on the web to show all information managed over the DTG file, effective driving management of vehicles can be achieved. In addition to this, by periodically transferring various information to a server over a mobile telecommunication system - the speed depending on driving distance, alarm services, automatic notice messages based on $\mathrm{G}$ when accidents have happened, individual job management using SMS, monitoring of vehicles using location information, guidance about arrivals of vehicles, and safety management information of vehicles (speed, location information, RPM, failures, emergencies) - services such as the detection of speeding cars, non-driving cars, idling cars, and vehicles for break time violation can be supported in real time.[1,2]

As clients of the proposed framework, a company of middle- or large-scale taxis and trucks may be become. If the requests of those companies occur, we can quickly support the adapted application services based on the framework. Also, clients can quickly have an advantage of launching this system at low cost.

The advantages and differentiation of the proposed framework are as follows. Existing systems based on simple location information only provide functions of monitoring for the current location and past tracking path, and geo-fence functions. But, the proposed system based on a framework can provide the existing functions as well as functions of advanced query processing. In other words, it is possible to use advanced functions such as shown searching for close gas stations with low cost, searching for the nearest taxi, analyzing a user's driving pattern and proposing a guide for economical driving, proposing a possible driving path to access guests", delivering service that can be saved, proposing a driving path for delivery cars, proposing a time table for car maintenance, and so on.

\section{System Design}

In this chapter, we present the architecture for the collection of status information of vehicles, the GPS mechanism for driving and tracking of vehicles, and the software architecture for the proposed system. The status information of vehicles is collected by a CPU connecting a CAN driver IC interface with a CAN interface of an OBD connector based on SPI. It is shown in Figure $1[4,5]$.

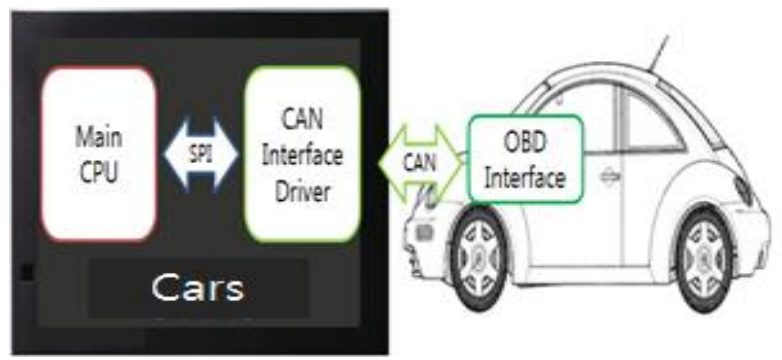

Figure 1. Architecture for Collection of Status Information of Vehicles

The status information needed for the safe driving of vehicles, such as speed, acceleration, status information of vehicles, are collected over the CAN interface of the terminal devices of vehicles. The collected information is transferred to a central monitoring server through an LTE gate, and the status and driving information of vehicles are analyzed over a central monitoring server $[8,9]$. 


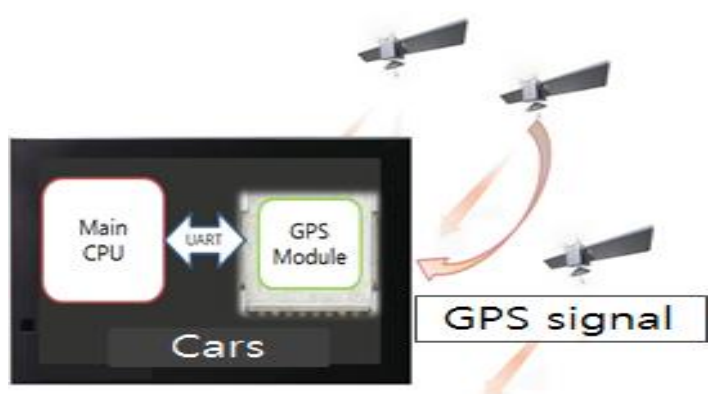

\section{Figure 2. Architecture of GPS Mechanism for Driving and Tracking of Vehicles}

Generally, for data about driving and the tracking of vehicles, the time and location information of vehicles based on GPS are transferred to a server. Figure 2 shows the architecture of the GPS mechanism the driving and tracking of vehicles.

\subsection{Middleware of the Integrated Monitoring System}

Information about the location, status, recorded video images, and still images of vehicles are transferred to remote servers by the terminal devices of vehicles over LTE communication networks. For the proposed system, a Hybrid Cloud Data Management System (HCDMS) comprising a Hadoop and DBMS was used. After analyzing information transferred from the terminal devices of vehicles, the data of location, status, video images, and still images are stored in an HBase based on Hadoop. Other information such as information about users, vehicles, and meta information for system management are stored in RDBMS [8, 9].

Also, a large amount of location information received from LTE terminals is indexed in distributed multi-nodes by an index module of the distributed location information of HCDMS. In order to process various users' queries based on location information (K-nn query processing, query processing for range, skyline query processing, continuous query processing, and so on), Mapreduce was used by a time-space query processing module based on the distributed location information of HCDMS [7-9].

This section should describe the concepts or the methodologies for solving a scientific questions or problems. Authors can change the section title.

\subsection{Adapted API}

An API of a monitoring system for vehicles is supported to develop an adapted application service for users. It can be applied to develop applications (fleet management, call-taxi services, and management services for delivery vehicles) based on various platforms (PC, web, and smart devices) [5].

Data processed by the monitoring platform of vehicles are collected from LTE terminals and are designed to be processed.

Those data are as follows:

- Current location and speed of vehicles

- Number of engine rotations and torque

- Signal of brake and accelerator pedal

- Status of gear

- Rate of fuel consumption per hour

- Current and average fuel consumption

- Driving distance 
- Cooling water, temperature of oil and engine

- Information of exhaust for $\mathrm{CO} 2$

- Information of desorption and attachment,

Information of diagnostics for failure

- Image recorded by terminal devices,

- Video images

Because the data collected from all terminal devices will be very large, in order to process a large amount of data, we developed a platform in a distributed cloud environment. The developed platform may be provided for clients as a type of an API, and then clients can support adapted applications or can provide an adapted application as a type of service for users [10-12].

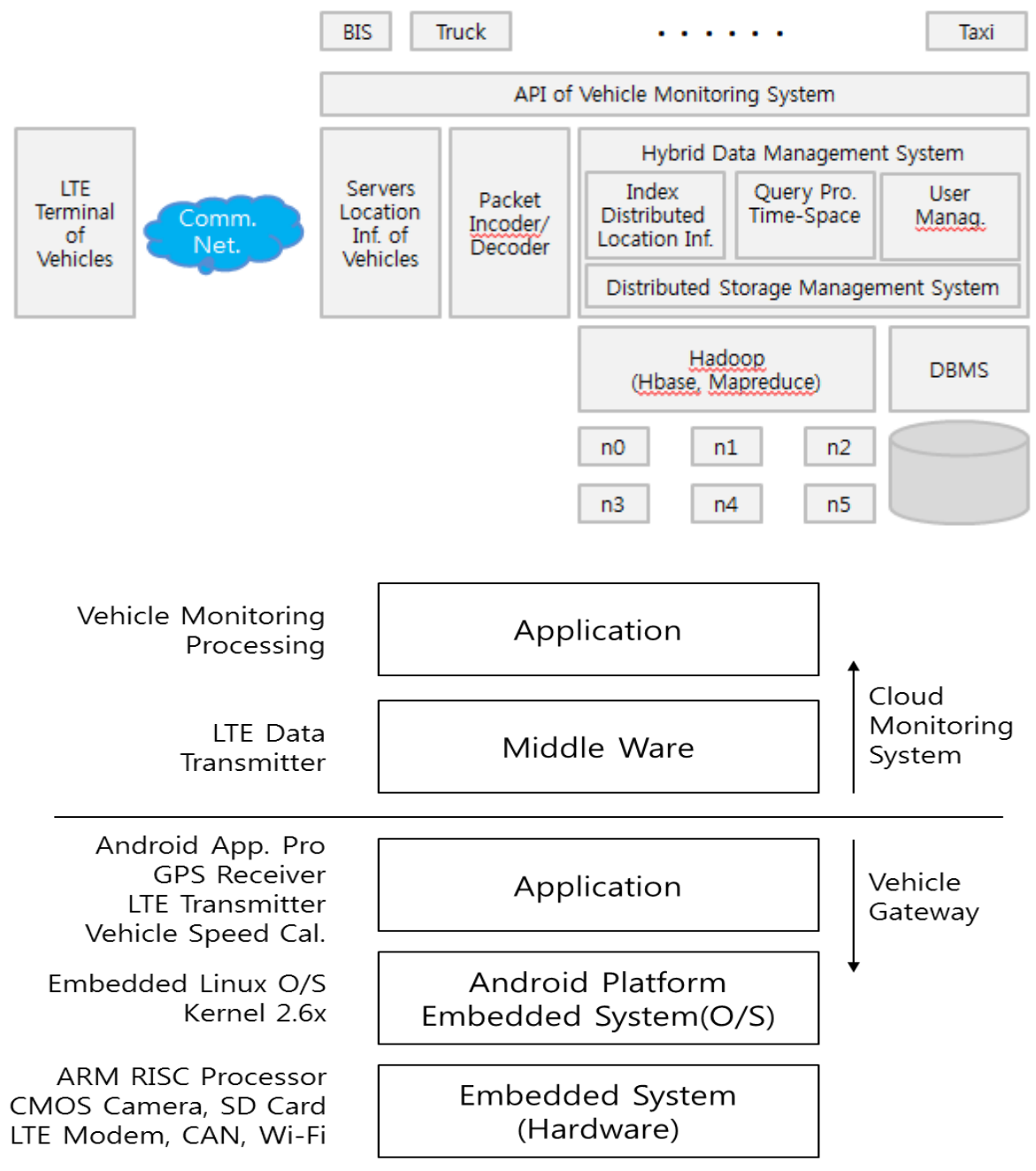

Figure 3. Software Architecture of Monitoring System

The functions of the API are:

- Create/Delete for the database of RDBMS

- Create/Delete for the database of HBase

- Configuration for database schema of vehicle monitoring

- Registration/Cancel for terminal devices 
- Configuration/Change for transmission cycle of terminal devices

- Configuration/Change for types of transmission data of terminal devices

- K-NN/Range/Skyline Query Processing

- Registration/Change/Cancel for continuous query processing

- Registration/Cancel for query about failure diagnostics

- Registration/Cancel for query of vehicle inspection

- Registration/Cancel for query about monitoring of Vehicle's path secession

- Query for creating cluster of vehicle's trajectory

- Query for proposition of driving path to save fuel

- Registration / Change/Cancel for events

- Registration / Change/Cancel for clients

- Configuration types of clients (Windows, Web, Android, iOS)

- Configuration / Change for UI template depending on types of clients

- Sending messages to terminal devices

- Function of expansion of API depending on a user's request

In order to store the management data, we support a Postgres over RDBMS and overcome the limitations for development and commercialization. Also, as a storage system to store the data of location, images, and video images, HBase and HDFS of Hadoop are applied.

We are not simply monitoring and storing information about vehicles at a monitoring center. According to advances of the terminal devices of a vehicle, various algorithms are adapted in the Safe Management System of vehicles, which applies an interactive communication function between a centralized monitoring center and the terminal devices of vehicles. Also, it is equipped with a function of transmission in real time [5, 6].

For example of guide services for vehicles, if the request signal about driving paths occurs to vehicles over a monitoring system, the response message is transferred to servers by terminal devices of vehicles depending on request events. After forecasting the driving path of a vehicle in servers, if the current driving path is straight, servers do not transfer location information of the vehicle to the terminal device of the vehicle. Then, information of the current location automatically calculated by the server is adapted.

For cases of changes in the driving path, the proposed system was designed to transfer and store information of the current location of a vehicle to a server. As a result, it can reduce the amount of traffic. The development environment uses Linux 2.6xx as the operating with Java, HTML5, and Javascript as languages.

The Figure 3 ware showed the software architecture of monitoring system and the internal functions for gateway system of vehicles.

\section{Conclusion}

Monitoring systems for the location of vehicles has already been developed and sold by many domestic and foreign companies. But, existing solutions or products are customized for clients in a restricted manner and are then deployed depending on the client's infrastructure. Also, they have high cost and take a long time for the initial launching of solutions or products. In addition, the types of supported services are simply monitoring of the location for vehicles or retrieving the past moving path, and so on. To overcome these problems, we designed and implemented a platform based on clouds. We can obtain advantages to quickly support adapted services and to reduce cost for the initial launching of solutions and the amount of time to develop applications. Also, by analyzing the path of current driving based on analyzing the path of past driving, we could design and implement a platform system that applies an algorithm to search for the best path of driving. 


\section{Acknowledgements}

The research was supported by a grant from the Academic Research Program of Korea National University of Transportation in 2013.

\section{References}

[1] D.-Y. Kim, G.-K. Park, N. Quoc Tuan and B.-T. Moon, "Implementation of an Intelligent Identification System for Ship's Abnormal Navigation using Fuzzy Inference", Proceedings of KIIS Fall Conference 2011, vol. 21, no. 2, (2011), pp. 29-30.

[2] D.-Y. Kim, G.-K. Park, J.-S. Jeong and G.-U. Kim, "Implementation of an Intelligent System for Identifying Abnormal Navigating Ships", Korean Institute of Intelligent Systems, vol. 22, no. 1, (2012), pp. 75-80.

[3] K.-M. Bae, G.-Y. Park, D.-J. Lee, J.-S. Lim, S.-R. Lee and C.-Y. Lee, "Development of Tank Lorry Monitoring System for Safety Using Intelligent Computing Device", Journal of the Korean Institute of Gas, vol. 12, no. 2, (2008) June, pp. $42-47$.

[4] W. J. Barbiaux, T. D. McCarthy, S. D. Bromley and G. W. Milliorn, "Vehicle monitoring arrangement and system, US Patent, (1989).

[5] A. Goel and V. Gruhn, "A fleet Monitoring System for Advanced Tracking of Commercial Vehicles", Proceedings of the 2006 IEEE International Conference on Systems, Man, and Cybernetics (SMC 2006), Taipei, Taiwan, (2006) August 10, pp. 2517-2522.

[6] N. Bressan, L. Bazzaco, N. Bui, P. Casari, L. Vangelista and M. Zorzi, "The Deployment of a Smart Monitoring System Using Wireless Sensor and Actuator Networks", Smart Grid Communications (SmartGridComm), 2010 First IEEE International Conference, USA, (2010) October 4-6.

[7] Hadoop. http:hadoop.apache.org.

[8] J. Park, B. Hong and C. Ban, "A Query Index for Continuous Queries on RFID Streaming Data", Science in China Series F: Information Sciences, vol. 51, no. 12, (2008), pp. 2047-2061.

[9] F. Chang, J. Dean, S. Ghemawat, W. C. Hsieh, D. A. Wallach, M. Burrows, T. Chandra, A. Fikes and R. E. Gruber, "Bigtable: A Distributed Storage System for Structured Data", ACM Transactions on Computer Systems (TOCS) TOCS, Article No. 4, vol. 26, no. 2, (2008) June.

[10] C.-Y. Lee, H.-K. Kim, K.-H. Ko, J.-W. Kim and H.-C. Jeong, "A VoIP Traffic Monitoring System based on NetFlow v9", IJAST, vol. 4, (2009) March, pp. 1-8.

[11] J. Woo, "Information Retrieval Architecture for Heterogeneous Big Data on Situation Awareness", IJAST, vol. 59, (2013) October, pp. 113-122, October 2013.

[12] H. Park, Y. Kwak and S. Song, "Design of the Communication Unit and Monitoring System Based on 4G", Journal of Advanced Information Technology and Convergence, vol. 3, no. 1, (2013), pp. 55-60.

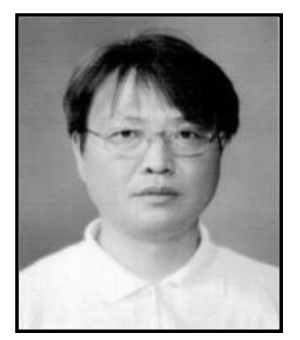

\section{Authors}

Hwase Park, he received a B.S. and M.S degree from Kyunghee University, Suwon, Korea, in 1987, 1989, and a Ph.D degree from Mowon university, Taejon, Korea, in 2011 Currently he is an professor in the Department of Electronic Communications, Daelim Collage University, Anyang Korea. His research interest includes Digital Communication System and Embedded System.

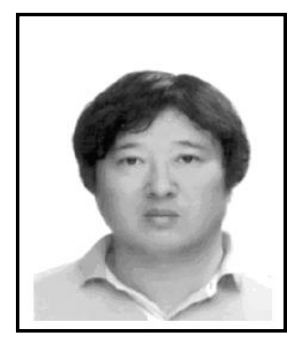

Seokil Song, he received B.S., M.S., and Ph.D. degrees in computer and communication engineering from Chungbuk National University in 1998, 2000, and 2003, respectively. Currently, he is an associate professor in the Department of Computer Engineering at Korea National University of Transportation, Chungbuk, Korea. His research interests include database management systems, concurrency control, high dimensional index structures, storage systems, moving object database, sensor network, and XML database. 


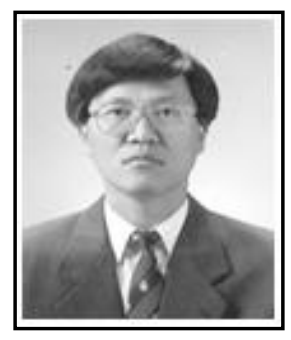

Yoonsik Kwak, received his B.S. degree in Electrical Engineering from the University of Cheongju in 1984, his M.S.E.E. degree from the University of Kyunghee in 1986 and his $\mathrm{Ph} . \mathrm{D}$. degree from the University of Kyunghee in 1994. He worked at Korea National University of Transportation in the Department of Computer Engineering and rose to the level of Full Professor. His research interests are in the areas of signal processing, Internet communication, microcomputer system, and applications of these methods to mobile system. Corresponding Author

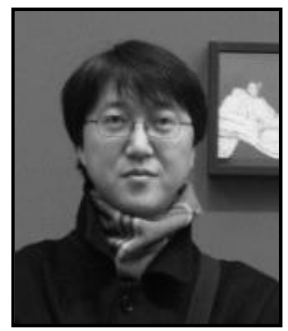

Youngsik Kwak, he received a B.B.A. degree from Sungkyunkwan University, Seoul, Korea, in 1990, an MBA. degree from Sungkyunkwan University, Seoul, Korea, in 1994, a M.S. degree from Texas Tech University, Lubbock, TX, in 1997, and a Ph. D. degree from Sungkyunkwan University, Seoul, Korea in 1999, in marketing. He had been a marketing consultant for Daewoo Economic Research Institutes from 1999 to 2002. Currently he is an associate professor in the Department of Venture and Business, Gyeongnam National University of Science and Technology, Jinju, Korea. His research interests include pricing on- and off-line. 
International Journal of Control and Automation

Vol.8, No.3 (2015) 